\title{
Primary Dural Closure for Retrosigmoid Approaches
}

\author{
Garrett T. Venable ${ }^{1} \quad$ Mallory L. Roberts ${ }^{1} \quad$ Ryan P. Lee $^{1} \quad$ L. Madison Michael, $I^{2,3}$ \\ ${ }^{1}$ College of Medicine, University of Tennessee Health Science Center, \\ Memphis, Tennessee, United States \\ 2 Department of Neurosurgery, University of Tennessee Health \\ Science Center, Memphis, Tennessee, United States \\ ${ }^{3}$ Semmes-Murphey Neurologic \& Spine Institute, Memphis, \\ Tennessee, United States \\ J Neurol Surg B 2018;79:330-334.

\begin{abstract}
Address for correspondence L. Madison Michael, II, MD, SemmesMemphis, TN 38120, United States (e-mail: mmichael@semmes-murphey.com).
\end{abstract} \\ Murphey Neurologic \& Spine Institute, 6325 Humphreys Boulevard,
}

\begin{abstract}
\section{Keywords}

- retrosigmoid craniotomy

- retrosigmoid approach

- primary dural closure

- CSF leak

Object Primary closure of posterior fossa dura can be challenging, and postoperative cerebrospinal fluid (CSF) leaks continue to represent a common complication of the retrosigmoid approach. We describe a simple technique to allow for primary closure of the dura following retrosigmoid approaches. The incidence of CSF leaks using this method is reported.

Methods A retrospective chart review was conducted on all cases of retrosigmoid craniotomies performed by the senior surgeon from February 2009 to February 2015. The primary outcome was development of postoperative CSF leak or pseudomeningocele. Length of stay, lesion type, and other surgical complications were also reported. Results Eighty-six patients underwent a retrosigmoid craniotomy during the study period. The most common indications for retrosigmoid craniotomy were microvascular decompression (58\%) and tumor resection (36\%). No allo- or autografts to repair the dural defect were needed, and no lumbar drains were used. No patients developed CSF otorrhea, rhinorrhea, or incisional leak postoperatively.

Conclusion Primary dural closure is possible in retrosigmoid approaches without the use of allo- or autografts and may prevent postoperative CSF leaks when combined with other posterior fossa closure techniques. Careful attention to the handling of the dural flap is necessary to achieve this.
\end{abstract}

\section{Introduction}

In skull base neurosurgery, the retrosigmoid approach has been widely described and utilized for access to various pathologies of the posterior fossa and the cerebellopontine angle. ${ }^{1-8}$ Despite the widespread utilization of this approach, one significant complication remains frustratingly common: postoperative cerebrospinal fluid (CSF) leak form the inner ear, nares, or the surgical site itself with varying incidence $(0-22 \%){ }^{7,9,10}$ In a 2004 pooled-group analysis of 2,273 patients from 14 studies, Selesnick et al. reported an overall CSF leak rate of $10.6 \%$ after retrosigmoid surgeries. ${ }^{11}$

received

September 2, 2016 accepted after revision July 10, 2017 published online

November 10, 2017
Many techniques have been described to reduce rates of postoperative CSF leak after retrosigmoid craniotomy, including meticulous dural closure in single or multiple layers with bony reconstruction, the use of varying allografts (fascial, fat, and or muscle autografts), postoperative lumbar drainage, and pressure or vacuum dressings with varying degrees of success. $^{1,7,10,12-14}$ Two series have reported the incidence of postoperative CSF leak following retrosigmoid craniotomy without the use of allo- or autograft. In 1999, Gal and Bartels reported an incidence of $2.9 \%$ with only the use of bone wax, and Yamakami et al reported an incidence of $4 \%$ with a similar technique in 2004. ${ }^{15,16}$
License terms Stuttgart · New York

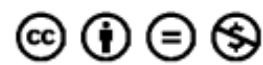


In this study, we report our experience in utilizing a technique to maintain the integrity of the dural flap, allowing for primary dural closure from the retrosigmoid approach. We report the incidence of postoperative CSF otorrhea, rhinorrhea, and incisional leak or pseudomeningocele. The technical nuances of primary dural closure of the retrosigmoid approach are described within the manuscript.

\section{Methods}

A retrospective chart review was performed to identify the primary surgeon's (L. Madison Michael) patients who underwent retrosigmoid craniotomy from February 2009 to February 2015. Electronic medical records-including discharge summary, history and physical, operative and radiographic magnetic resonance imaging (MRI) and computed tomography (CT) reports, and clinic notes-were reviewed to determine the diagnosis of each patient and to confirm each surgical approach with particular emphasis on development of CSF rhinorrhea, otorrhea, pseudomeningocele, or incisional leak by the first surgical follow-up. Patients were excluded if they did not undergo a retrosigmoid craniotomy. Individuals performing the chart review were all trained by one individual (Garrett T. Venable) to ensure consistency of review and coding. To further verify accuracy, two individuals (Garrett T. Venable and L. Madison Michael) reviewed all patient records.

We collected the following data points for each study patient: (1) age at the time of surgery, (2) primary diagnosis, (3) sex, (4) time to first post-surgical follow-up, (5) presence or absence of postoperative CSF leak or pseudomeningocele, (6) length of hospital stay (LOS), and (7) any other complications of surgery (e.g., meningitis or wound infection).

Our primary outcome measure was development of a CSF leak (otorrhea, rhinorrhea, or incisional leak) during the initial hospital stay or by the first surgical follow-up. The institutional review boards of Methodist Le Bonheur Healthcare and the University of Tennessee Health Sciences Center approved this study. All statistics were calculated using SPSS v.22 (IBM, Armonk, New York).

\section{Results}

\section{Case Series}

Eighty-six patients were identified who underwent retrosigmoid craniotomy between February 2009 and February 2015. Patient characteristics can be found in - Table 1 . Median age at the time of surgery was 55 years (range, 21-80 years) with 53 (61.6\%) females and 33 (24.8\%) males. The most common indications for retrosigmoid craniotomy were microvascular decompressions $(50,58.1 \%)$ and tumor resection $(31,36 \%)$. Primary dural closure was possible in all cases, and no patients required allo- or autografts to repair the dural defect. Median LOS was 3 days (range, 1-33 days). Median time to first postoperative follow-up was 24 days (range, 12-679 days), and 5 (5.8\%) patients were lost to follow-up. No patients developed a CSF leak (otorrhea, rhinorrhea, or incisional leak) or pseudomeningocele by
Table 1 Characteristics of patients undergoing retrosigmoid craniotomy

\begin{tabular}{|l|l|}
\hline Variable & Value \\
\hline Age, years; median, range & $55,21-80$ \\
\hline Gender & \\
\hline Male & $33(38.4 \%)$ \\
\hline Female & $53(61.6 \%)$ \\
\hline Indication & $50(58.1 \%)$ \\
\hline Microvascular decompression & $31(36 \%)$ \\
\hline Tumor & $7(8.1 \%)$ \\
\hline Acoustic neuroma & $7(8.1 \%)$ \\
\hline Metastasis & $7(8.1 \%)$ \\
\hline Meningioma & $3(3.5 \%)$ \\
\hline Glioma & $3(3.5 \%)$ \\
\hline Vestibular schwannoma & $3(3.5 \%)$ \\
\hline Hemangioblastoma & $1(1.2 \%)$ \\
\hline Chordoma & $2(2.3 \%)$ \\
\hline Dermoid cyst & $2(2.3 \%)$ \\
\hline $\begin{array}{l}\text { Brainstem cavernous } \\
\text { malformation }\end{array}$ & $1(1.2 \%)$ \\
\hline $\begin{array}{l}\text { Cerebellar abscess } \\
\text { Length of stay, days; median (range) }\end{array}$ & $3,1.2-33.1$ \\
\hline Time to first follow-up, days; \\
median, range
\end{tabular}

hospital discharge or at the first postoperative follow-up visit. One (1.3\%) patient developed a postoperative wound hematoma that resolved without surgical intervention. No patients developed a postoperative infection.

\section{Surgical Technique}

Scalp incisions vary depending on pathology. The incision for microvascular decompression surgery is linear and is $5 \mathrm{~cm}$ in length. It is deepened to expose the bone, and no attempt is made to develop soft tissue layers. For all other pathologies, the incision is C-shaped and retroauricular. The galea and skin are then carried forward as the first layer followed by mobilization of the underlying muscles inferiorly and that of the musculoperiosteal layer superiorly. In all cases, craniectomies are performed, and the posterior aspect of the transverse and/or sigmoid sinus is exposed (-Fig. 1). If mastoid air cells are visualized, bone wax or bone paste is used to seal them to exclude communication with the middle ear. The dura is opened immediately posterior and inferior to the sigmoid and transverse venous sinuses, respectively, (-Fig. 2), and stay sutures are placed on the dural edge to enhance exposure (-Fig. $\mathbf{3}$ ). The posterior fossa dura maintains its position on the moist surface of the cerebellum, and a wet cottonoid is placed on the top of the dura to prevent drying (-Fig. 4). Intermittent irrigation of the cottonoid and dura is performed throughout the case as necessary to avoid desiccation. Primary closure of the dura begins inferiorly and 


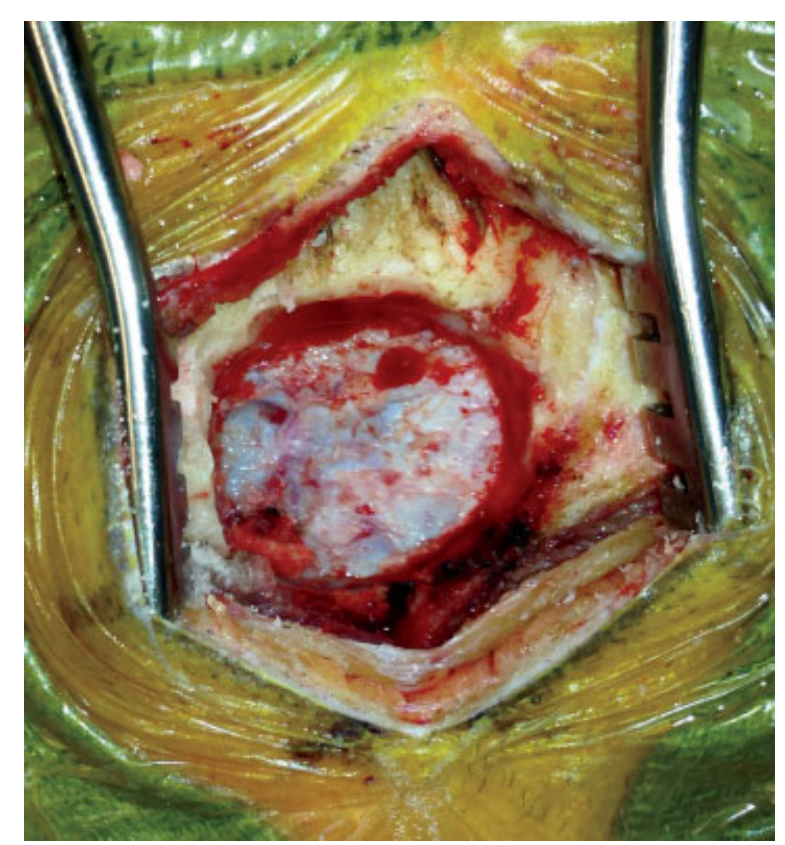

Fig. 1 Bony exposure of a typical microvascular decompression case. The distal transverse sinus and proximal sigmoid sinus are visualized.

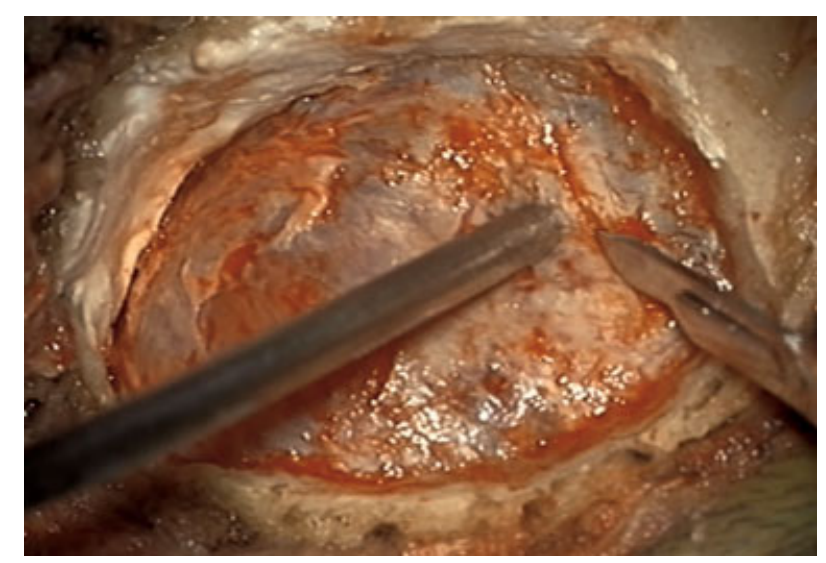

Fig. 2 The dura is initially incised along the inferior border of the transverse sinus. It is then carried inferiorly just posterior to the sigmoid sinus.

is carried superiorly using interrupted $4-0$ Nurolon (Ethicon) sutures (-Fig. 5). Complete closure was possible in all patients. DuraSeal (Integra) tissue glue is then injected over the suture line. A dry piece of Gelfoam (Pfizer) is placed within the epidural space, and contoured titanium mesh is used to reconstitute the bony defect ( - Fig. $\mathbf{6}$ ). No fat, lumbar drain, or head dressing is used. The wound is closed in layers. The skin is closed in a running fashion using 3-0 Rapide (Ethicon) suture. Dermabond (Ethicon) is placed overlying the incision as the sole dressing.

\section{Discussion}

Postoperative CSF leak following retrosigmoid craniectomies remains a frustrating complication and represents a large economic burden to patients and hospital systems. In a 2004 cost analysis of postoperative CSF leaks and cost effectiveness

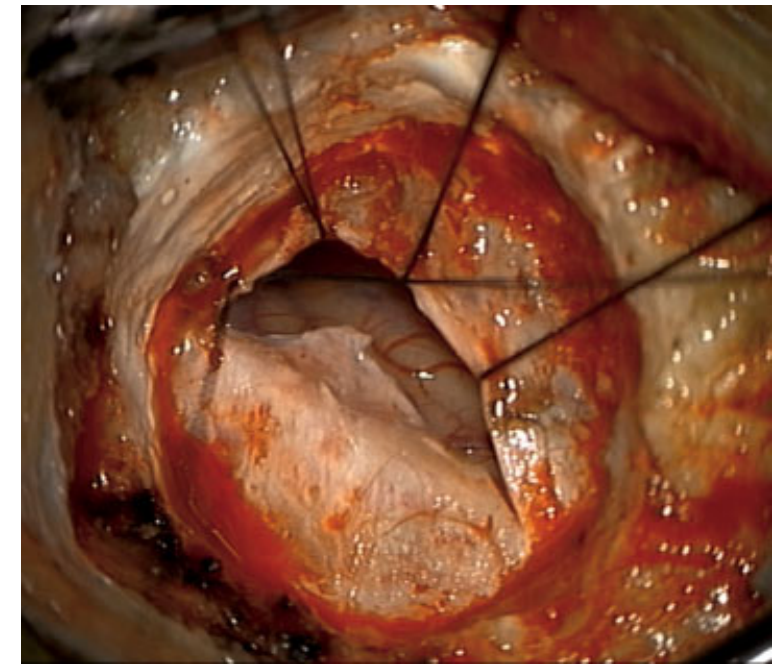

Fig. 3 Stay sutures are placed along the venous sinus side of the dural opening.

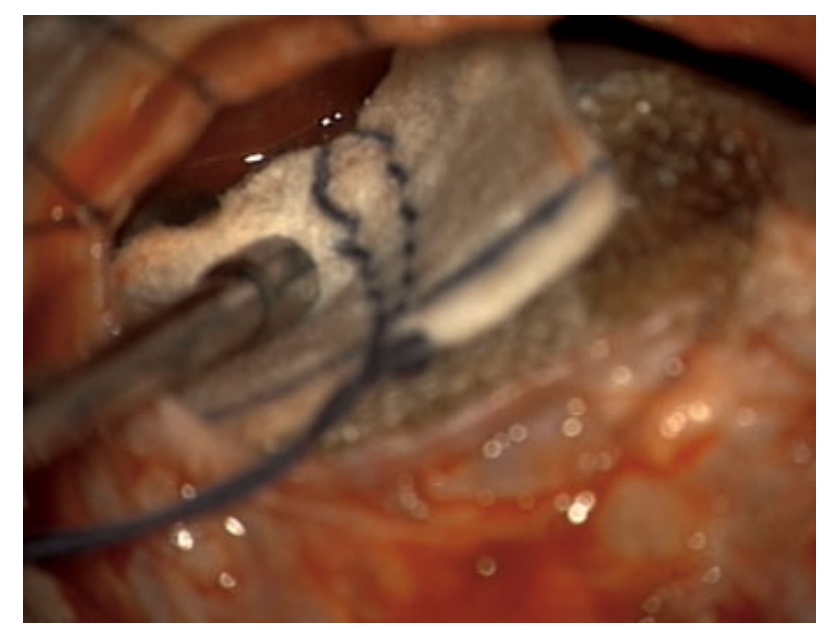

Fig. 4 The dural flap is left directly on the moist surface of the cerebellum and is covered by a moist cotton patty.

of dural sealants, Grotenhuis found that 44 (10.7\%) of 412 total patients who experienced postoperative CSF leaks accrued $21.7 \%$ of the total cost for the group and $€ 17,412-$ or $\$ 19,088$ -more per procedure. ${ }^{17} \mathrm{~A}$ more recent study by Hendricks et al found the average cost of readmission for postoperative CSF leak after endoscopic transsphenoidal surgery to be $\$ 24,613$; 18 additionally, patients with elevated body mass index (BMI) may have an increased risk for CSF leak. ${ }^{6}$ Many techniques addressing the problem of CSF leaks have been described through the years with varying rates of success, including galeal, fascial, or fat grafts alone or in combination $7,10,19,20$; meticulous primary closure of anatomical layers ${ }^{9,15,16}$; dural sealants ${ }^{21}$; dural allografts ${ }^{22}$; bone cement reconstruction ${ }^{5,14}$; postoperative lumbar drainage $e^{2,19,23,24}$; and postoperative compression dressing. ${ }^{10,20}$ Two recent studies have reported $0 \%$ CSF leak rates. Ling et al describe their closing technique during retrosigmoid craniectomies, which included autologous fat graft overlying the primary dural closure (with or without the use of a dural allograft patch), Medpor Titan 


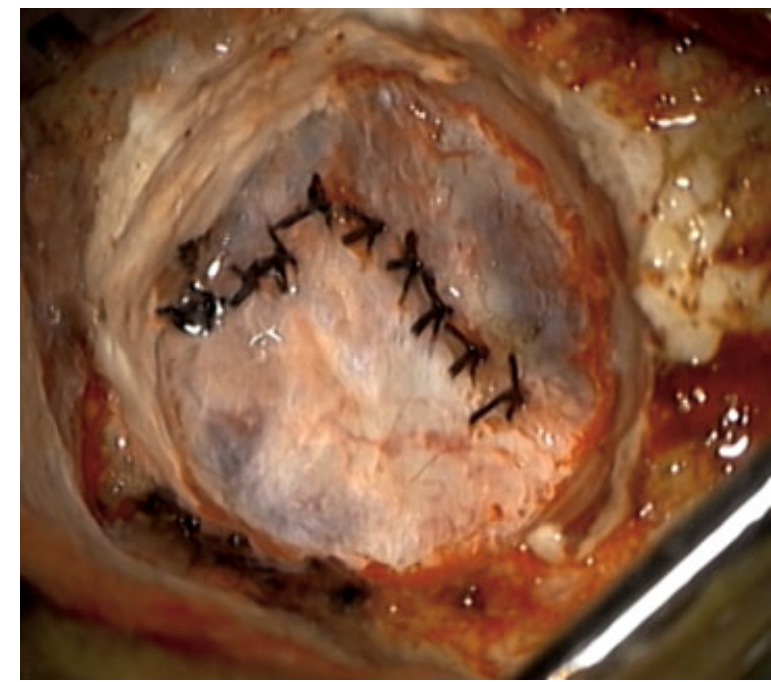

Fig. 5 Primary closure of a microvascular decompression case is demonstrated here.

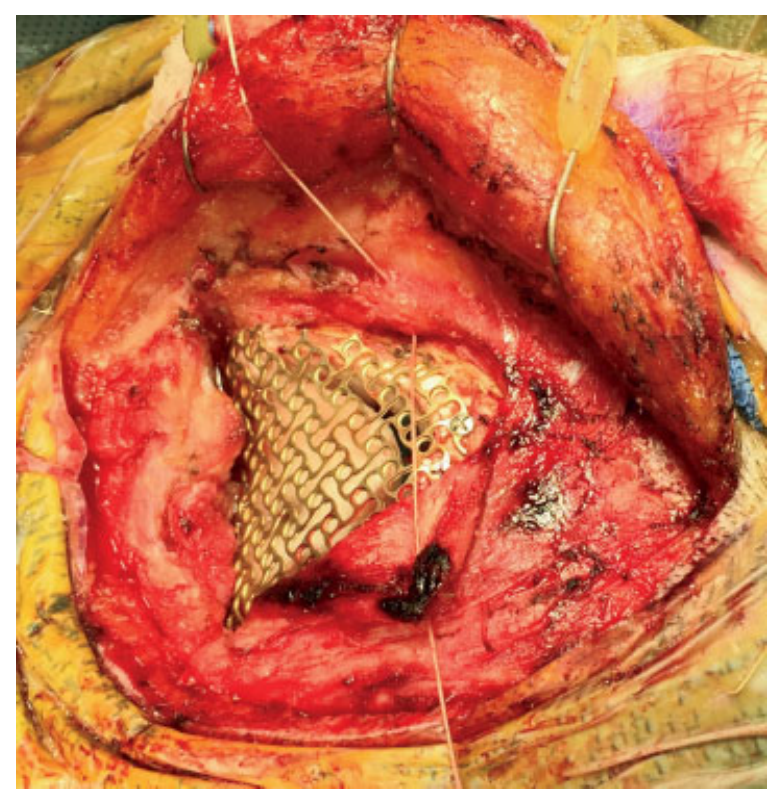

Fig. 6 Reconstruction of the bony defect is performed using titanium mesh.

cranioplasty, and 1 to 2 days of postoperative pressure dressing. Their mean LOS was 3.8 days with no reported complications at a median follow-up of 1 year. ${ }^{10}$ Eseoneu at al report a 0\% CSF leak when using calcium phosphate cement cranioplasty as well as a statistically significant decrease in CSF leaks as compared with polyethylene titanium mesh cranioplasty ( $0 \%$ vs $4.5 \%, p=0.03) .{ }^{10,14}$

Traditional teachings involving the retrosigmoid approach recommend opening the dura along the periphery of the craniotomy/craniectomy and carrying it toward the venous sinus. ${ }^{25}$ In comparison with the dura adjacent to the venous sinuses, the dura overlying the cerebellum is quite thin and friable; folding the dura upon itself or retracting the dural leaflets with stay sutures with this technique can more easily lead to dehydration and retraction of the delicate dural flap as the case progresses. Primary closure of the dura, then, can be difficult in this situation, necessitating additional measures to mitigate postoperative CSF leaks. To ensure primary closure of the dura in cases involving the retrosigmoid approach, we have found it necessary to shift the dural incision much closer to the venous sinuses. In all cases, exposure of the posterior aspect of the venous sinus is performed as it reduces the need for cerebellar retraction. To ensure that venous sinus injury does not occur during the exposure, we prefer the use of a craniectomy for better exposure. With direct visualization of the venous sinus, it is possible to open the dura at the point of maximum thickness. Retraction sutures are placed through the dural edge on the venous sinus side in an effort to maximize visualization, reduce cerebellar retraction, and avoid rundown of blood from the epidural space. Avoiding the use of retraction sutures or folding of the dura on the cerebellar side allows the layer to remain moist throughout the procedure. A wet cottonoid prevents the outer layer from becoming dehydrated by the light of the microscope. Following the completion of the intradural portion of the procedure, the dura is re-approximated using interrupted sutures. Interrupted sutures-as opposed to a running suture-are felt to lead to a watertight closure, as supported by the work of Megyesi et al. ${ }^{26}$ DuraSeal is used to reinforce the suture line and is in no way a substitute for incomplete dural closure. It is biodegradable and prevents the patient from undergoing a possible second incision to obtain fat autograft. Dry Gelfoam is placed in the epidural space to protect the dura from muscle attachment during the healing process, which may lead to postoperative headaches. Reconstruction of the cranial defect is accomplished using titanium mesh. Advantages of the mesh, aside from producing an excellent cosmetic result, include its inert properties, ease of implantation, and absence of artifact on postoperative imaging. There is also no concern of degradation over the years when using the titanium mesh, as is the case with calcium phosphate cement when there is incomplete osteogenesis. ${ }^{27}$

Although we did not evaluate each component of our closure technique independently, many of them have been validated by the neurosurgical literature. ${ }^{1,9,15,16,21,26}$ We believe that it is important to understand each of these closure techniques, but a primary dural closure should be the goal in all cases. It is a simple and effective technique and serves as the first step in mitigating CSF leaks after retrosigmoid craniectomies.

In addition to its benefits in reducing CSF leaks, our closure is efficient and economical. There are no time-consuming additional steps, such as the harvesting of autograft (i.e., fat, fascia, galea, etc.) or placement of a lumbar drain. Each of these additional time-consuming steps also has an associated cost. In 2005, it was estimated that 1 minute of operating room time could cost as much as $\$ 133$, depending on the procedure type, and is likely a low estimate today. ${ }^{28}$ Costs are also generated with each cranioplasty technique and with the use of dural sealants and dural allografts; however, it is important to remember that each technique is utilized to prevent readmissions for CSF leak, which cost nearly $\$ 25,000$. We believe our technique minimizes the economic burden while providing maximal benefit to each patient. 


\section{Conclusions}

Primary dural closure is possible in retrosigmoid approaches without the use of dural allo- or autografts. Careful attention to the handling of the dural flap is necessary to achieve this. This may help obviate the need for graft placement, complete cranioplasty, or postoperative lumbar drain placement when combined with other well-known closure techniques.

\section{Note}

No part of this paper has been published or previously presented.

\section{Conflict of Interest}

The authors report no conflicts of interest concerning the materials or methods used in this study or the findings specified in this paper.

\section{Acknowledgments}

The authors received no material or financial sources of support for this manuscript. The authors wish to thank Andrew J. Gienapp (Department of Medical Education, Methodist University Hospital, Memphis, Tennessee and Department of Neurosurgery, University of Tennessee Health Science Center, Memphis, Tennessee) for technical and copyediting, preparation of the manuscript and figures for publishing, and publication assistance with this manuscript.

\section{References}

1 Hardy DG, Macfarlane R, Moffat DA. Wound closure after acoustic neuroma surgery. Br J Neurosurg 1993;7(02):171-174

2 Brennan JW, Rowed DW, Nedzelski JM, Chen JM. Cerebrospinal fluid leak after acoustic neuroma surgery: influence of tumor size and surgical approach on incidence and response to treatment. J Neurosurg 2001;94(02):217-223

3 Becker SS, Jackler RK, Pitts LH. Cerebrospinal fluid leak after acoustic neuroma surgery: a comparison of the translabyrinthine, middle fossa, and retrosigmoid approaches. Otol Neurotol 2003; 24(01):107-112

4 Ansari SF, Terry C, Cohen-Gadol AA. Surgery for vestibular schwannomas: a systematic review of complications by approach. Neurosurg Focus 2012;33(03):E14

5 Baird CJ, Hdeib A, Suk I, et al. Reduction of cerebrospinal fluid rhinorrhea after vestibular schwannoma surgery by reconstruction of the drilled porus acusticus with hydroxyapatite bone cement. J Neurosurg 2007;107(02):347-351

6 Copeland WR, Mallory GW, Neff BA, Driscoll CL, Link MJ. Are there modifiable risk factors to prevent a cerebrospinal fluid leak following vestibular schwannoma surgery? J Neurosurg 2015; 122(02):312-316

7 Fishman AJ, Marrinan MS, Golfinos JG, Cohen NL, Roland JT Jr. Prevention and management of cerebrospinal fluid leak following vestibular schwannoma surgery. Laryngoscope 2004;114(03): 501-505

8 Fishman AJ, Hoffman RA, Roland JT Jr, Lebowitz RA, Cohen NL. Cerebrospinal fluid drainage in the management of CSF leak following acoustic neuroma surgery. Laryngoscope 1996; 106(08):1002-1004

9 Cueva RA, Mastrodimos B. Approach design and closure techniques to minimize cerebrospinal fluid leak after cerebellopontine angle tumor surgery. Otol Neurotol 2005;26(06):1176-1181
10 Ling PY, Mendelson ZS, Reddy RK, Jyung RW, Liu JK. Reconstruction after retrosigmoid approaches using autologous fat graftassisted Medpor Titan cranioplasty: assessment of postoperative cerebrospinal fluid leaks and headaches in 60 cases. Acta Neurochir (Wien) 2014;156(10):1879-1888

11 Selesnick SH, Liu JC, Jen A, Newman J. The incidence of cerebrospinal fluid leak after vestibular schwannoma surgery. Otol Neurotol 2004;25(03):387-393

12 Selesnick SH, Liu JC, Jen A, Carew JF. Management options for cerebrospinal fluid leak after vestibular schwannoma surgery and introduction of an innovative treatment. Otol Neurotol 2004; 25(04):580-586

13 Della Pepa GM, Montano N, Lucantoni C, Alexandre AM, Papacci F, Meglio M. Craniotomy repair with the retrosigmoid approach: the impact on quality of life of meticulous reconstruction of anatomical layers. Acta Neurochir (Wien) 2011;153(11): 2255-2258

14 Eseonu CI, Goodwin CR, Zhou X, et al. Reduced CSF leak in complete calvarial reconstructions of microvascular decompression craniectomies using calcium phosphate cement. J Neurosurg 2015;123(06):1476-1479

15 Gal TJ, Bartels LJ. Use of bone wax in the prevention of cerebrospinal fluid fistula in acoustic neuroma surgery. Laryngoscope 1999;109(01):167-169

16 Yamakami I, Uchino Y, Kobayashi E, Yamaura A, Oka N. Removal of large acoustic neurinomas (vestibular schwannomas) by the retrosigmoid approach with no mortality and minimal morbidity. J Neurol Neurosurg Psychiatry 2004;75(03):453-458

17 Grotenhuis JA. Costs of postoperative cerebrospinal fluid leakage: 1-year, retrospective analysis of 412 consecutive nontrauma cases. Surg Neurol 2005;64(06):490-493, discussion 493-494

18 Hendricks BL, Shikary TA, Zimmer LA. Causes for 30-day readmission following transsphenoidal surgery. Otolaryngol Head Neck Surg 2016 Feb;154(02):359-365. doi: 10.1177/0194599815617130

$19 \mathrm{Wu} \mathrm{H}$, Kalamarides M, Garem HE, Rey A, Sterkers O. Comparison of different wound closure techniques in translabyrinthine acoustic neuroma surgery. Skull Base Surg 1999;9(04):239-242

20 Leonetti J, Anderson D, Marzo S, Moynihan G. Cerebrospinal fluid fistula after transtemporal skull base surgery. Otolaryngol Head Neck Surg 2001;124(05):511-514

21 Schiariti M, Acerbi F, Broggi M, et al. Two alternative dural sealing techniques in posterior fossa surgery: (polylactide-co-glycolide) self-adhesive resorbable membrane versus polyethylene glycol hydrogel. Surg Neurol Int 2014;5:171

22 Abla AA, Link T, Fusco D, Wilson DA, Sonntag VK. Comparison of dural grafts in Chiari decompression surgery: review of the literature. J Craniovertebr Junction Spine 2010;1(01):29-37

23 Bayazit YA, Celenk F, Duzlu M, Goksu N. Management of cerebrospinal fluid leak following retrosigmoid posterior cranial fossa surgery. ORL J Otorhinolaryngol Relat Spec 2009;71(06): 329-333

24 Nonaka Y, Fukushima T, Watanabe K, et al. Contemporary surgical management of vestibular schwannomas: analysis of complications and lessons learned over the past decade. Neurosurgery 2013;72(2 Suppl Operative)ons103-ons115, discussion ons115

25 Quiñones-Hinojosa A, Schmidek HH. Schmidek \& Sweet Operative Neurosurgical Techniques: Indications, Methods, and Results. 6th ed. Philadelphia, PA: Elsevier/Saunders; 2012

26 Megyesi JF, Ranger A, MacDonald W, Del Maestro RF. Suturing technique and the integrity of dural closures: an in vitro study. Neurosurgery 2004;55(04):950-954, discussion 954-955

27 An J, Liao H, Kucko NW, et al. Long-term evaluation of the degradation behavior of three apatite-forming calcium phosphate cements. J Biomed Mater Res A 2016;104(05):1072-1081

28 Macario A. What does one minute of operating room time cost? J Clin Anesth 2010;22(04):233-236 